\title{
Variabilité clonale de l'infradensité chez Quercus petraea Premiers résultats obtenus sur boutures d'un an
}

\author{
Gérard NEPVEU \\ I.N.R.A., Station de Recherches sur la Qualité des Bois, \\ Centre de Recherches forestieres de Nancy, Champenoux, F 54280 Seichamps
}

\begin{abstract}
Résumé
Les variabilités clonales de l'infradensité du bois ainsi que des caractéristiques de vigueur et de forme sont estimées sur des individus d'un an de chêne rouvre obtenus par bouturage de rejets de souche. L'héritabilité de l'infradensité calculée par analyse de variance dans les ramets est très forte $(0,65)$ avec une variabilité génotypique élevée $\left(463 \mathrm{à} 575 \mathrm{~g} / \mathrm{dm}^{3}\right.$ entre les moyennes de clones extrêmes). Cependant, la liaison ortet-ramet (calculće sur un échantillon réduit) ne semble pas significative.

Les conséquences de ces résultats pour un programme d'amélioration par la voie végélative portant notamment sur la densité du bois, sont discutées.
\end{abstract}

Depuis plusieurs années, un programme d'amélioration génétique est engagé en France sur le Chêne rouvre (Quercus petraea) et le Chêne pédonculé (Quercus robur). Son objectif est d'essayer de produire des génotypes alliant une bonne qualité intrinsèque du bois à une croissance relativement rapide. Précisons que par bonne qualité, il faut entendre aptitude à des usages nobles tel le tranchage, pour lequel il convient de rechercher des bois tendres et de faibles retraits.

Ces deux objectifs - qualité et vigueur - semblaient antagoniques puisque depuis toujours les forestiers observent que les chênes à accroissements larges ne peuvent être utilisés en tranchage, d'où l'application dans les chênaies de qualité d'une sylviculture très prudente conduisant à des cernes fins donc à des révolutions très longues - 200 à 300 ans — pour atteindre des diamètres d'exploitabilité convenables.

Or, la Station de Recherches sur la Qualité des Bois du C.N.R.F. a montré que quelques individus présentaient des accroissements larges et une bonne qualité ; cette découverte rejoint d'ailleurs des observations empiriques sur les chênes des vallées de la Saône et de l'Adour encore que, dans ces deux cas, il s'agisse de peuplements 
entiers, d'où la possibilité d'un effet des provenances ou des milieux très particuliers dont il est question.

L'intérêt qu'il y aurait à produire rapidement des chênes de bonne qualité explique l'importance de la connaissance du contrôle héréditaire de cette aptitude, condition sine qua non pour qu'un programme de sélection individuelle puisse être envisagé. Des recherches s'avèrent d'autant plus nécessaires qu'à notre connaissance aucune référence n'existe sur ce sujet dans la littérature spécialisée à l'exception du travail de Polge, Lemoine \& Deret (1977) qui, il faut le préciser, n’a étudié la variabilité génétique qu’au niveau infraspécifique.

La voie végétative a été envisagée pour multiplier les arbres de qualité voulue ; ce choix repose entre autres sur la mise au point récente du bouturage herbacé de rejets de souche de chêne (Garbaye et al., 1975 ; Cornu et al., 1977 ; Galbaye et al., 1977 ; KaZANDJIAN, 1977). C"est la raison pour laquelle nous nous intéresserons à la variabilité génotypique des caractères, c'est-à-dire la variabilité de l'ensemble des effets des gènes de l'individu (effets additifs mais également effets de dominance et d'épistasie).

La densité du bois étant une composante décisive de la qualité chez le chêne par suite de ses liaisons positives avec la dureté et le retrait du bois (Polge \& Keller, 1973, op. cit.), l'étude dont il est rendu compte ici a précisément pour objet de fournir des indications sur le contrôle génétique précoce de ce caractère. Signalons que les liaisons juvénile - adulte très significatives observées chez le chêne pour la densité du bois - sur le plan phénotypique et avec un bois juvénile un peu plus âgé il est vrai - nous encouragent dans cette voie (Courtorsier, 1976; Mourey, 1979); sur le matériel dont un échantillon a fourni les ramets que nous étudions, le premier de ces auteurs a trouvé une corrélation juvénile-adulte de 0,520 significative au seuil de $1 \%$ (161 degrés de liberté).

D'autres données, vigueur ou forme des très jeunes arbres, ont été prises en considération. A leur égard, plusieurs objectifs sont possibles : on peut s'en servir comme prédicteurs de l'état adulte, ce qui est difficile puisque l'on n'a pas d'indication sur leurs liaisons juvénile-adulte; on peut aussi envisager de sélectionner pour les caractères jeunes eux-mêmes afin d'économiser sur les travaux de dégagement et de mise en forme des jeunes sujets. Dans cette perspective, les corrélations avec le critère de qualité retenu, la densité du bois, ont été calculées afin de voir si l'élimination de clones peu vigoureux ou mal conformés pourrait faire disparaître des génotypes précieux sur le plan de la qualité intrinsèque du bois.

Notre préoccupation est donc d'étudier les possibilités offertes par une sélection très précoce pour la qualité du bois, laquelle revêt, comme on l'a dit, une importance considérable chez le chêne. Cela dit, nous sommes conscients d'un problème que nous évoquerons au cours de la relation de cette expérience, à savoir l'impossibilité de séparer les effets «souche» (conditions de milieu entourant la souche, état physiologique de celle-ci pouvant avoir par exemple des conséquences sur l'aptitude au bouturage de certains clones) des effets génétiques dans les différences entre clones, sauf dans certaines conditions. Cet effet «souche» s'apparente à ce que nous pourrons appeler un effet «maternel» que, dans le domaine de la reproduction sexuée, les améliorateurs forestiers connaissent bien, à propos par exemple de la croissance juvénile souvent liée au poids de la graine; celui-ci augmente en effet artificiellement la variance génétique de celle-là. Au sujet de la densité du bois en particulier, on 
pourrait penser que le cambium des rejets, dérivant de celui de l'ortet (arbre aduite), va engendrer un plan ligneux semblable. Dans ce cas précis cependant, on peut s interroger sur la frontière entre effet «maternel» et effet du code génétique des cellules du cambium.

Nous pouvons nous trouver devant deux situations :

1) Variance génotypique ( + celle des effets «maternels») nulle statistiquement; dans ce cas, on sait que de toute façon il faut abandonner l'espoir d'une sélection très précoce à moins que les effets génétiques et «maternels» ne soient pas additifs mais « interactifs» ce qui laisse entière la question.

2) Variance génotypique ( + celle des effets «maternels») significative; dans ce cas, des espoirs de sélection précoce sont permis, espoirs que l'on pourra concrétiser en expérimentant de façon à apprécier la part des effets de variabilité due aux effets «maternels».

Nous avons donc pris le parti de tirer le maximum d'informations du précieux matériel disponible afin de savoir si d'emblée, il fallait renoncer à la sélection précoce (situation 1) ou si quelque possibilité existait de la mettre en cuvre (situation 2).

\section{I. - Matériel et méthode}

Au cours de la saison de végétation 1977 des rejets herbacés ont été prélevés sur une quarantaine de souches de Quercus petraea récemment abattus en coupe de régénération avec des âges variant de 150 à plus de 200 ans, dans les forêts de Bride et de Saint Jean (département de la Moselle). Aucune sélection phénotypique n’a été pratiquée sur les arbres-mères, si ce n'est le fait que les arbres n'ayant pas rejeté de souche ont été bien évidemment éliminés. De même, les clones dont les rejets ne se sont pas ou très peu enracinés n'ont pu être pris en compte. Les rejets se sont enracinés sur tourbe non fertilisée, en serre, sous un brouillard artificiel. Ils ont été ensuite transplantés dans des pots, et maintenus sous serre jusqu'à la fin de l'expérience. Au printemps 1978, ces rejets ont débourré produisant une ou plusieurs pousses. Du point de vue de la nature du végétal et du milieu de croissance, nous avons donc des conditions radicalement différentes pour les ortets et les ramets. Nous verrons que ce fait pourra contribuer à expliquer certains résultats.

\subsection{Mesure des caractères de vigueur et de forme}

Sur la plus grande de ces pousses, on a noté dès la fin de la saison de végétation 1978, son diamètre à la base, sa longueur, son écart angulaire avec la verticale. Le nombre de pousses de chaque ramet a été également relevé. Nous appellerons ces deux derniers caractères : caractères de forme pour simplifier, même si cela est un peu abusif.

Les mesures d'angle n'ont pu être effectuées sur les rejets trop peu vigoureux car les pousses étaient trop petites. Aussi, dans le souci de ne pas trop biaiser la 



hiver $1978-79$

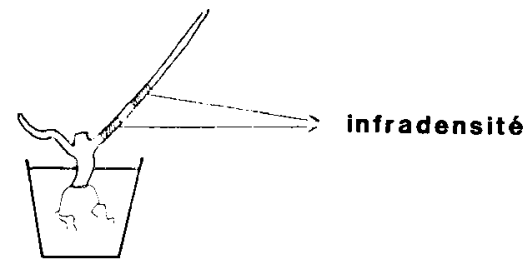

FIGURE 1

Matériel végétal utilisé pour estimer les paramètres génétiques sur les boutures d'un an Material obtainment. Measures

Saison $1977: 1977$ season.

Fin saison $1978:$ End of 1978 season.

Hiver 1978-1979: 1978-1979 winter.

Infradensité : basic density.

Largeur de la pousse la plus longue : length of the longest shoot.

Diamètre de la pousse la plus longue : diameter of the longest shoot.

Ecart angulaire avec la verticale de la pousse la plus longue : angular deviation with the vertical of the longest shoot.

Nombre de pousses par ramet (ici $\mathrm{n}=2$ ) : shoot number per ramet (here $n=2$ ). 
variabilité des caractères autres que l'écart angulaire avec la verticale, a-t-on constitué deux échantillonnages, le second étant extrait du premier :

- le premier compte 28 clones, 7 ramets par clone sur lequel on calculera les variabilités clonales pour le nombre de pousses, la longueur et le diamètre de la plus grande pousse, ainsi que les corrélations entre ces trois caractères ;

- le second compte 25 clones, 7 ramets par clone; on pourra calculer sur ce groupe d'échantillons, la variance clonale pour l'angle de la pousse ainsi que ses liaisons avec les trois autres caractéristiques de vigueur.

\subsection{Mesure de la densité du bois}

A la fin de l'hiver 1978-1979, les pousses ont été coupées. A la base de chacune d'entre elles, on a prélevé deux tronçons de $5 \mathrm{~mm}$ à $2 \mathrm{~cm}$ de longueur, sans nœud, qui ont été soigneusement écorcés. C'est l'infradensité qui a été mesurée, par la méthode de saturation intégrale (KEYLWERTH, 1954) ; le poids saturé a été obtenu par ébullition durant 48 heures, l'état anhydre par séjour de 24 heures dans une étuve à $102^{\circ}$.

On a délibérément choisi de prendre deux éprouvettes par pousse afin de contrôler la variabilité des mesures à l'intérieur d'une pousse, variabilité incluant les erreurs expérimentales qui pouvaient être importantes vu le poids très faible de ces éprouvettes (avec des poids anhydres parfois inférieurs à deux centièmes de gramme). En outre, les longueurs des tronçons de pousse étant différentes $(5 \mathrm{~mm}$ à $2 \mathrm{~cm})$ on pouvait craindre que leur saturation soit plus ou moins complète, autre source d'erreur dans le calcul des infradensités.

La mesure des infradensités a été effectuée sur 19 clones extraits de l'échantillon de 25 clones cité plus haut, représentés chacun par 10 ramets, l'infradensité de chaque pousse étant mesurée sur deux éprouvettes (soit $19 \times 10 \times 2=380$ échantillons).

Il est à noter que les caractères de vigueur ou de forme d'une part, l'infradensité d'autre part, n'ayant pu être mesurés sur les mêmes ramets, les liaisons entre ces deux groupes de données ne seront calculées qu'au niveau moyenne de clone.

La figure 1 résume les étapes de l'obtention du matériel ainsi que les mesures effectuées.

\section{II. - Résultats}

\subsection{Vigueur et forme}

\subsection{Variabilité clonale}

Le tableau 1 présente les résultats des analyses de variance de l'effet «clone». L'héritabilité est calculée par la formule :

avec :

$$
\mathbf{h}^{2}=\frac{\sigma^{2}{ }_{\mathrm{cl}}}{\sigma_{\mathrm{cl}}^{2}+\sigma^{2}}=\frac{\sigma^{2}{ }^{2}{ }}{\sigma^{2}{ }_{p}}
$$

$\sigma^{2}$ el : variance entre clones ou variance génotypique.

$\sigma_{\mathrm{e}}^{2}$ : variance entre ramets dans le clone.

$\sigma^{2}{ }_{\mathrm{el}}+\sigma_{\mathrm{e}}^{2}=\sigma_{\mathrm{p}}^{2}$ : variance totale (ou phénotypique) 
où $\sigma^{2}$ el et $\sigma^{2}$ esont estimés grâce aux carrés moyens de l'analyse de variance, le carré moyen «ramet dans clone» estimant $\sigma^{2}{ }_{e}$ et le carré moyen "clone » estimant $\sigma^{2}{ }_{e}+\mathrm{n} \sigma_{\mathrm{cl}}(\mathrm{n}=$ nombre de ramets par clone $)$.

\section{TABleav 1}

Analyse de variance pour l'effet clone : caractères de vigueur et de forme Variance analysis for clonal effect : vigour and shape characteristics

\begin{tabular}{|c|c|c|c|c|c|c|}
\hline Caractère & Moyenne & $\begin{array}{c}\text { Carré } \\
\text { moyen } \\
\text { clone }\end{array}$ & $\begin{array}{l}\text { Carré moyen } \\
\text { ramet dans } \\
\text { clone } \\
\text { (erreur) }\end{array}$ & $\begin{array}{c}F \\
\text { clone }\end{array}$ & $\begin{array}{l}\text { Degrés } \\
\text { de liberté }\end{array}$ & $\begin{array}{c}\text { Héritabilité } \\
\text { au sens } \\
\text { large }\end{array}$ \\
\hline $\begin{array}{c}\text { Nombre } \\
\text { de pousses } \\
\text { par ramet }=\mathrm{n}\end{array}$ & 2,05 & 1,9494 & 0,9337 & $2,09 * *$ & 27 et 168 & 0,13 \\
\hline $\begin{array}{l}\text { Longueur de } \\
\text { la plus grande } \\
\text { pousse }=\mathrm{L}\end{array}$ & $0.41 \mathrm{~m}$ & 0,3359 & 0,0319 & $10,52^{* *}$ & 27 et 168 & 0,58 \\
\hline $\begin{array}{c}\text { Diamètre } \\
\text { de la pousse } \\
\text { la plus longue }=\varnothing\end{array}$ & $0,63 \mathrm{~cm}$ & 0,3123 & 0,0213 & $14,63 * *$ & 27 et 168 & 0,66 \\
\hline $\begin{array}{c}\text { Ecart angulaire } \\
\text { avec la verticale } \\
\text { de la pousse } \\
\text { la plus longue }=\alpha\end{array}$ & $29,7^{\circ}$ & 498 & 279 & $1,78^{*}$ & 24 et 150 & 0,10 \\
\hline
\end{tabular}

Caractère : characteristic.

Moyenne : mean.

Carré moyen «clone » : «clone » mean square.

Carré moyen "ramet dans clone» (erreur) : «ramet in clone» mean square (error).

Degrés de liberté : degrees of freedom.

Héritabilité au sens large : broad sense heritability.

* Effet clone significatif au seuil de 5 p. 100. Clonal effect significant at $5 \mathrm{p} .100$ level.

** Effet clone significatif au seuil de 1 p. 100. Clonal effect significant at 1 p. 100 level.

Nous notons un effet clone extrêmement important pour la croissance alors que pour le nombre de pousses et l'écart angulaire avec la verticale, deux caractères décrivant la forme des ramets, les valeurs de $\mathrm{F}$ ne sont pas très éloignées des seuils de signification. Ce dernier point est à rapprocher des résultats de KLEINSCHMIT, OTto \& SAuer (1975) sur des clones greffés de Quercus robur et Quercus petraea installés dans six dispositifs différents, résultats qui indiquent que le contrôle génétique semble faible sur la forme du chêne, telle qu'ils l'avaient définie. 
Pour connaître, dans la variance « clone », la part des effets proprement génétiques et celle des effets que nous avons appelés « maternels » dans l'introduction, il faudrait poursuivre l'expérience plusieurs années en réitérant les mesures : les effets maternels diminuant en général avec l'âge, on devrait assister à une baisse de la variance «clone », pour le cas bien sûr où ces effets sont significatifs. On pourrait également bouturer plusieurs fois chaque pousse et observer si la variance «clone» décroît avec le rang des générations : un test en cours réalisé avec les mêmes clones que dans la présente étude mais avec des boutures de $2^{\circ}$ génération devrait nous éclairer prochainement sur cette question. Signalons en outre que nous y individualiserons les boutures de $1^{\text {re }}$ génération afin de connaître la part de variabilité intraclone imputable à celle-ci, variabilité qu'on peut également attribuer à des effets «maternels» même s'ils ne sont pas de la même nature que ceux évoqués ici (effets «souche»).

La figure 2 donne les courbes de fréquence des moyennes de clone pour les quatre caractères. On constate une fois de plus la très grande variabilité clonale pour la longueur et le diamètre de la plus grande pousse $(8$ à $83 \mathrm{~cm} ; 3$ à $10 \mathrm{~mm}$ respectivement). La valeur moyenne élevée de l'angle de la pousse la plus longue est assez inquiétante : près de $30^{\circ}$. On peut en concevoir quelque appréhension pour l'avenir de la forme des plants bouturés d'autant que ce caractère, comme on l'a vu, semble difficile à corriger par la voie génétique (héritabilité faible). Le tuteurage pose des problèmes sur le plan pratique. La taille ou le recépage peuvent être une solution ainsi que nous l'indiquent certains essais en cours, pour donner aux jeunes arbres une forme convenable. On peut aussi espérer rétablir l'orthotropie en multipliant les générations de bouturage.
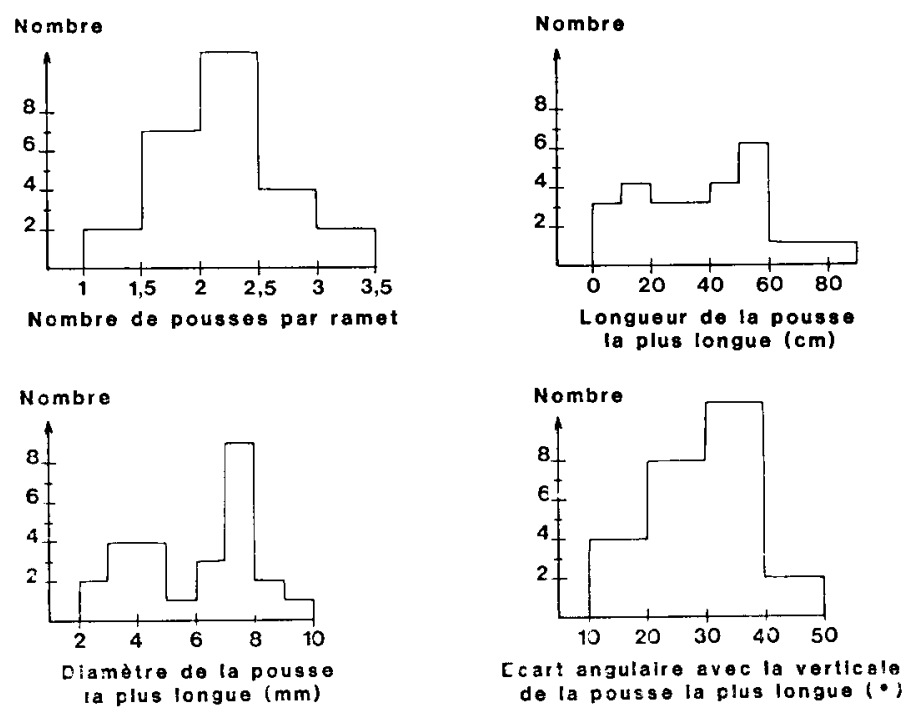

FIGURE 2

Courbes de fréquence des moyennes de clones pour quelques caractères

Histograms of clonal means for some characteristics 


\subsection{Densité du bois}

L'analyse de variance pour l'infradensité va prendre en considération les variabilités «clone», « ramet dans clone» et «éprouvette dans ramet» (terme d'erreur). Une analyse hiérarchique est donc indiquée.

\section{TABLEAU 2}

Analyse de variance pour l'effet clone : infradensité en $\mathrm{g} / \mathrm{dm}^{3}$ (moyenne: $522 \mathrm{~g} / \mathrm{dm}^{3}$ )

Variance analysis for clonal effect : basic density in $\mathrm{g} / \mathrm{dm}^{3}$ (mean : $522 \mathrm{~g} / \mathrm{dm}^{3}$ )

\begin{tabular}{|c|c|c|c|c|}
\hline $\begin{array}{c}\text { Source } \\
\text { de variation }\end{array}$ & $\begin{array}{l}\text { Degré } \\
\text { de liberté }\end{array}$ & $\begin{array}{l}\text { Carré } \\
\text { moyen }\end{array}$ & $\begin{array}{c}\mathrm{F} \\
\text { calculé }\end{array}$ & Estimation des carrés moyens \\
\hline Clone........ & 18 & 19425 & $22,10^{* *}$ & $\sigma^{2}{ }^{2}+k_{22} \sigma^{2} r+k_{3} \sigma^{2}{ }_{1}$ \\
\hline Ramet dans clone .. & 171 & 879 & $7,33^{* *}$ & $\sigma^{2}{ }_{0}+k_{1} \sigma^{2}{ }_{r}$ \\
\hline $\begin{array}{l}\text { Eprouvette dans ra- } \\
\text { met (erreur) .... }\end{array}$ & 190 & 120 & & $\sigma^{2}{ }_{e}$ \\
\hline Total ... & 379 & & & \\
\hline
\end{tabular}

Source de variation : source of variation.

F calculé : $F$ calculated.

Estimation des car rés moyens : mean square estimation.

Ramet dans clone : ramet in clone.

Eprouvette dans ramet : sample in ramet.

Avec :

$$
\begin{array}{ll}
\sigma^{2}{ }_{c l} & : \text { variance «clone } . \\
\sigma^{2}{ }_{r} & : \text { variance « ramet dans clone } . \\
\sigma^{2}{ }_{\mathrm{e}} & : \text { variance «éprouvette dans ramet }, \\
\sigma^{2}{ }_{r l}+\sigma_{r}^{2}++\sigma^{2}=\sigma_{\mathrm{e}}^{2}: \text { variance phénotypique, } \\
\mathrm{k}_{1}=\mathrm{k}_{{ }_{2}}=2: \text { nombre d'éprouvettes par ramet. } \\
\mathrm{k}_{3}=20: \text { nombre total d'éprouvettes par clone. }
\end{array}
$$

Les estimations de $\sigma^{2}{ }_{\mathrm{cl}}, \sigma_{\mathrm{r}}^{2}$ et $\sigma^{2}{ }^{2}$ à partir des carrés moyens sont respectivement égales à 927,380 et 120 (l'infradensité étant exprimée en $\mathrm{g} / \mathrm{dm}^{3}$ ), soit une

héritabilité $\frac{\sigma^{2}{ }^{2 l}}{\sigma^{2}{ }_{\mathrm{cl}}+\sigma_{\mathrm{r}}^{2}+\sigma_{\mathrm{c}}^{2}}$ égale à 0,65 .

Cette valeur élevée qui ne doit pas faire oublier les remarques concernant la possibilité d'existence d'effets «maternels», est intéressante pour l'améliorateur, d'autant plus que ce contrôle génétique étroit est associé à une variabilité importante : 463 à $575 \mathrm{~g} / \mathrm{dm}^{3}$ entre les deux clones extrêmes. 
Au crédit de la méthodologie, il faut porter la valeur extrêmement faible de la variance «éprouvette dans ramet »: 120 (soit un écart-type de $11 \mathrm{~g} / \mathrm{dm}^{3}$ ), ce qui est un bon résultat pour des éprouvettes d'aussi faible taille.

\subsection{Vigueur et forme}

\subsection{Liaisons entre caractères}

L'analyse de covariance a un facteur - qui se pratique de la même façon que l'analyse de variance - permet de décomposer la covariance phénotypique entre les caractères $\mathrm{X}$ et $\mathrm{Y}$ en ses deux composantes, la covariance "clone » (génotypique $\operatorname{cov}_{\mathrm{el}}$ ) et la covariance intraclone (covariance due au milieu, $\operatorname{cov}_{\mathrm{e}}$ ). On a en effet $\operatorname{cov}_{\mathrm{p}}(\mathrm{X}, \mathrm{Y})=\operatorname{cov}_{\mathrm{cl}}(\mathrm{X}, \mathrm{Y})+\operatorname{cov}_{\mathrm{e}}(\mathrm{X}, \mathrm{Y})$.

Les corrélations génotypique et due à l'environnement se déduisent des analyses de variance-covariance de la manière suivante :

corrélations génotypiques entre les caractères $\mathrm{X}$ et $\mathrm{Y}$ :

$$
r_{G}=\frac{\operatorname{cov}_{c l}(X, Y)}{\sqrt{\sigma^{2}{ }_{c l}(X) \times \sigma_{c l}^{2}(Y)}}
$$

corrélations dues au milieu entre les caractères $\mathrm{X}$ et $\mathrm{Y}$ :

$$
r_{E}=\frac{\operatorname{cov}_{\mathrm{e}}(X, Y)}{\sqrt{\sigma^{2}{ }_{\mathrm{e}}(\mathrm{X}) \times \sigma^{2}{ }_{\mathrm{e}}(\mathrm{Y})}}
$$

L'estimation de $r_{G}$ permet de savoir dans quel sens évolue le caractère $Y$ si l'on fait varier $X$ en changeant de clone; celle de $r_{\mathrm{F}}$ fournit une information de même ordre mais cette fois lorsque l'on modifie le milieu et non plus les génotypes. Les deux coefficients $\mathrm{r}_{G}$ et $\mathrm{r}_{\mathrm{E}}$ peuvent être tout à fait différents (PolgE \& ILLY, 1968 ; Polge, 1969 ; Nepveu, 1973) d'où l'intérêt de la décomposition de la covariance phénotypique $\operatorname{cov}_{\mathrm{p}}(\mathrm{X}, \mathrm{Y})$. Nous signalerons que des effets «maternels éventuels peuvent contribuer à biaiser nos estimations de $r_{G}$. Le tableau 3 donne les estimations de $r_{G}$ et $r_{E}$ pour différents couples de caractères :

\section{TABLEAU 3}

Liaisons entre différents caractères de vigueur et de forme

Relationships between various vigour and shape characteristics

\begin{tabular}{c|c|c}
\hline \hline Caractères & $\begin{array}{c}\text { Corrélation génotypique } \\
\mathrm{r}_{\mathrm{G}}\end{array}$ & $\begin{array}{c}\text { Corrélation due } \\
\text { au mileu } \mathrm{r}_{\mathbf{E}}\end{array}$ \\
\cline { 2 - 3 } $\mathrm{n}$ et $\mathrm{L}$ & $-0,039 \mathrm{NS}$ & $+0,049 \mathrm{NS}$ \\
$\mathrm{n}$ et $\varnothing$ & $+0,001 \mathrm{NS}$ & $+0,164 \mathrm{NS}$ \\
$\varnothing$ et $\mathrm{L}$ & $+0,888^{* *}$ & $+0,669^{* *}$ \\
$\alpha$ et $\mathrm{n}$ & $-0,038 \mathrm{NS}$ & $+0,140 \mathrm{NS}$ \\
$\alpha$ et $\mathrm{L}$ & $+0,347 \mathrm{NS}$ & $-0,101 \mathrm{NS}$ \\
$\alpha$ et $\varnothing$ & $+0,056 \mathrm{NS}$ & $-0,162^{*}$ \\
\hline
\end{tabular}

Corrélation génotypique $\mathrm{r}_{\mathrm{G}}$ : genotypic correlation $r_{G}$.

Corrélation due au milieu $\mathrm{r}_{\mathbf{F}}$ : environmental correlation $r_{n}$. 
A l'exception des liaisons significatives entre longueur et diamètre de la plus grande pousse qu'il était logique d'observer, les autres corrélations ne sont pas statistiquement différentes de zéro si ce n'est une valeur de $\mathrm{r}_{\mathbf{E}}$ négative (au demeurant proche du seuil à 5 p. 100) pour le couple écart angulaire avec la verticale $\times$ diamètre de la plus grande pousse qui suggérerait qu'à l'intérieur d'un clone les plants les plus gros soient les moins plagiotropes.

Il apparaît donc globalement que tant entre clones qu'à l'intérieur d'un même génotype, les caractères de forme (écart angulaire avec la verticale et nombre de pousses) sont indépendants de la vigueur. Ceci confirme les observations courantes.

\subsection{Densité du bois et caractères de vigueur et de forme}

Ainsi qu'il a été dit plus haut, seules les liaisons au niveau "moyennes de clone», qui s'apparentent d'assez près aux corrélations génotypiques, ont pu être calculées. Elles figurent sur le tableau 4.

\section{TABleau 4}

Liaisons entre l'infradensité du bois et les caractères de vigueur et de forme. Corrélations au niveau des moyennes de clone

Relationships between wood basic density and vigour and shape characteristics. Correlations between means of clone

\begin{tabular}{c|c|c}
\hline Liaison entre l'infradensité et & Valeur de $\mathrm{r}$ & Nombre de couples \\
\cline { 2 - 3 } $\mathrm{n}$ & $0,529^{*}$ & 20 \\
$\mathrm{~L}$ & $0,159 \mathrm{NS}$ & 20 \\
$\varnothing$ & $0,352 \mathrm{NS}$ & 20 \\
$\alpha$ & $-0,278 \mathrm{NS}$ & 20 \\
\hline
\end{tabular}

Liaisons entre l'infradensité et : relationships between basic density and

Valeur de $\mathrm{r}: r$ value.

Nombre de couples : number of pairs.

A l'exception d'une corrélation faiblement positive entre l'infradensité et le nombre de pousses par ramet, il n'apparaît pas de liaisons significatives. Ceci implique que le choix à ce stade de clones à bois peu dense n'aurait pas pour conséquence l'élimination des génotypes les plus vigoureux, ni de meilleure forme, encore qu'à notre avis la faible variabilité clonale pour l'écart angulaire avec la verticale de la pousse et le nombre de pousses par ramet n'aurait pas eu, même dans l'hypothèse où les clones présentant la meilleure qualité auraient été les plus mal conformés, de conséquences très fâcheuses. L'absence de liaison densité $\mathrm{x}$ vigueur nous écarte des schémas connus pour le chêne ; encore faut-il préciser que nous parlons ici, à très peu près, d'une liaison génotypique qui n'avait jamais été calculée, les corrélations obtenues jusqu'à présent restant au niveau phénotyique faute de dispositifs permettant l'étude de la source de variation génétique. 
Rappelons que le matériel dont il est question ici - pousses issues de boutures ayant passé une saison de végétation en serre - est extrêmement différent des arbres adultes de forêt naturelle que l'on étudie ordinairement : ceci pourrait expliquer des réponses du plan ligneux, donc de l'infradensité, à des variations de vigueur s'écartant sensiblement des règles connues sur des sujets en peuplement.

\subsection{Liaisons ortet-ramet pour l'infradensité du bois}

Une question vient immédiatement à l'esprit : le classement des clones pour la qualité correspond-il à celui des arbres dont ils sont issus par bouturage ? C'est la réponse à cette interrogation qui doit indiquer l'efficacité d'une sélection phénotypique pour la qualité en forêt.

A cet effet et malgré la taille faible de l'échantillon, nous avons mesuré l'infradensité de 13 ortets disponibles (arbres adultes) sur des coupes minces de carotte de sondage et ce, à la fois au voisinage de l'écorce et du cœur (pour la méthodologie, voir Courtoisier, 1976, op. cit.) ; les corrélations ortet-moyenne de ramet ont ensuite été calculées. Elles conduisent à des valeurs non significatives (seuil à $5 \%$ de 0,553 pour 13 couples) :

- $r=0,391$ avec l'infradensité côté cœur chez les ortets.

- $r=-0,094$ avec l'infradensité côté écorce chez les ortets.

On note que la valeur de la corrélation obtenue en prenant l'infradensité côté coeur pour l'ortet est plus forte que dans le deuxième cas. Cela suggérerait que, bien que le cambium en cause dans l'émission des rejets soit contemporain de celui fabriquant le bois côté écorce, le rajeunissement de son fonctionnement est tel que le bois formé dans les boutures de ces rejets se trouve beaucoup plus proche de celui de l'ortet jeune.

\section{III. - Discussion et conclusion}

L'un des résultats les plus importants de ce travail est la mise en évidence d'une variabilité clonale très forte pour la densité du bois et ce, sur les boutures ayant une saison de végétation seulement. Ceci pourrait inciter à l'optimisme si nous n'observions dans le même temps une liaison ortet-ramet non significative pour le caractère étudié ; ce dernier résultat indique que la sélection phénotypique en forêt d'arbres de faible densité ne se concrétise pas, au niveau des boutures d'une année qui en sont issues, par un gain génétique, preuve de son inefficacité, ce qui, il faut insister sur ce point, ne condamne pas cependant tout programme d'amélioration génétique. On pourrait en effet, sous réserve que la variabilité entre clones soit bien génétique, envisager une sélection pour la qualité sur test clonal et non plus en forêt; ceci serait naturellement plus lourd.

Cette contradiction pourrait s'expliquer par plusieurs phénomènes qui peuvent d'ailleurs intervenir simultanément :

1) Le sous-échantillon des 13 clones pour lesquels la liaison ortet-moyenne de ramets a été calculée présente une variabilité génétique plus petite que les 19 clones de l'échantillon complet ayant servi à l'estimation de l'héritabilité de la densité du 
bois. Cette hypothèse peut être écartée d'emblée puisqu'un nouveau calcul sur les 13 clones du sous-échantillon donne pour $\mathrm{h}^{2}$ une valeur de 0,74 (contre 0,65 pour l'échantillon complet).

2) La variabilité clonale observée au stade bouture n'est pas due à un effet proprement génétique mais à un effet «maternel». Nous avons déjà évoqué cette question et indiqué que l'étude de tests clonaux d'âge plus avancé ou bien de boutures de plusieurs générations pourrait nous éclairer.

3) Les génotypes ont des aptitudes différentes au bouturage qui interagissent par le biais d'une liaison génotype sur la qualité du bois masquant par là même les liaisons ortet-ramet. On conçoit aisément que cette aptitude au bouturage puisse être la cause de la très forte variabilité pour les caractères de vigueur. Cependant, si elle devait aussi expliquer la forte héritabilité de la densité du bois, on devrait observer une bonne corrélation vigueur-densité au niveau moyenne de clone, ce qui n'est pas le cas.

4) Les liaisons génotypiques juvénile-adulte entre bois d'un an et bois de $\mathbf{1 5 0}$ à 200 ans sont médiocres à la différence des bonnes corrélations phénotypiques observées chez le chêne (Courtoisier, 1976, op. cit.; Mourey, 1979, op. cit.). La valeur de la corrélation ortet-ramet plus élevée (mais cependant non significative) lorsque le bois de l'ortet est pris côté cœur n'est qu'une indication dans ce sens, à vérifier toutefois avec un meilleur échantillonnage.

5) Une forte interaction génotype $x$ environnement bouleverse le classement des génotypes dans les deux milieux extrêmement différents que sont une futaie parvenue à la coupe définitive et un substrat de tourbe sous nébulisation protégé par une serre. S'il en était ainsi, il faudrait craindre que la sélection de bons phénotypes pour plantation de leurs ramets dans des conditions de sylviculture différentes (par exemple culture d'arbres à grand espacement avec tailles) ne soit inutile. Cela nous obligerait à orienter la sélection en vue de la plantation dans ces types de milieux très artificialisés par des tests clonaux réalisés précisément avec cette sylviculture-ci.

6) L'échantillon de 13 couples ortet-ramet, même s'il comporte des individus à qualité $\mathrm{du}$ bois assez variable (cf. les différences clonales très importantes, 463 à $575 \mathrm{~g} / \mathrm{dm}^{3}$, pour la densité du bois), ne renferme pas de sujets très exceptionnels, rares par définition, à cernes larges et à bois cependant tendre, sujets que l'on cherche précisément à multiplier végétativement. Il se pourrait que, même si les liaisons ortet-ramet ne sont pas significatives sur la population globale, les quelques individus exceptionnels aient malgré cela des ramets croissant rapidement et à bois de bonne qualité. Cette faculté, inscrite dans un génotype très particulier, serait en quelque sorte le reflet, dans les ramets, du fait que les ortets correspondants échappent à la corrélation phénotypique défavorable largeur de cerne - densité du bois. Cela serait naturellement à vérifier avec un échantillonnage mieux adapté.

Il est à noter que l'effet clonal très fort observé sur la vigueur peut être, au même titre que la densité du bois, entaché d'effets maternels. Il faudra donc être également attentif à l'évolution de la variabilité «génotypique » de ce caractère avec l'âge. Les deux autres caractères qui concourent à décrire la forme de la tige quant à eux semblent beaucoup moins dépendants de la source de variation considérée. Terminons en rappelant que l'élimination précoce de clones peu vigoureux ou mal conformés n'a pas d'incidence sur la densité du bois. 
Cette étude qui n'est que le préliminaire d'un programme d'amélioration important a été entreprise malgré les réserves que l'on a signalées quant au matériel, afin de tirer parti au maximum de l'information fournie par les boutures issues de rejets de souches dont, à notre connaissance, on mesurait la variabilité de la densité pour la première fois.

Des expériences en cours actuellement au C.N.R.F. devraient apporter des éléments de réponse aux hypothèses formulées ici. Citons notamment :

- l'étude de plusieurs tests clonaux à des âges variables allant de 1 ou 2 ans à plus de 20 ans; il s'agit, dans ce dernier cas, de clones greffés mis à notre disposition par des forestiers allemands et sur lesquels nous étudierons des caractéristiques importantes autres que la densité, telles la rétractabilité du bois,

- l'utilisation dans les tests clonaux de boutures de plusieurs générations,

- des calculs de régression ortet-ramet en utilisant des ramets relativement âgés, comportant entre autres des boutures de chênes à accroissements larges et bois tendre.

Des tests clonaux sont également prévus.

Reçu pour publication le 10 juillet 1981.

\section{Summary}

Clonal variability of basic density in Quercus petraea.

First results in one-year-old cuttings

The clonal variabilities of wood basic density, vigour and shape characteristics are estimated in one-year-old individuals of Quercus petraea obtained by cutting of stock shoots. The heritability calculated by variance analysis in ramets is very high $(0.65)$ with a great genotypical variability (463 to $575 \mathrm{~g} / \mathrm{dm}^{3}$ between the extreme clonal means). However, the correlation between ortet and ramet (calculated in small sampling) does not seem significant.

The consequences of these results for a breeding programme with vegetative propagation are discussed, particularly for wood density.

\section{Zusammenfassung}

Klonvariabilität der Raumdichte der Traubeneiche. Erste Ergebnisse bei einjährigen Stecklingen

Die Klonvariabilitäten der Holzraumdichte so wie der Form- und Krafteigenschaften sind bei einjährigen, durch Stecklingsplanzung der Steubbendprosse gewonnenen Traubeneichen geschätzt.

Die Erblichkeit der aumdichte, durch Varianzanalyse in den Rameten gerechnet, ist sehr stark $(0,65)$ und die Variabilität des Genotypus ist sehr hoch (von 463 bis $575 \mathrm{~g} / \mathrm{dm}^{3}$ zwischen den extremen Mittelwerten der Klonen.

Jedoch ist die Beziehung Ausgangpflanze-Ramet (in einer kleineren Strichprobe) anscheinend nicht significativ.

Die Folgen dieser Ergebnisse werden diskutiert in Hinsicht auf ein vegetatives Züchtungsprogramm, insbesondere in Bezug auf Holzdichte. 


\section{Références bibliographiques}

Cornu D., Delran S., Garbaye J., Le Tacon F., 1977. Recherche des meilleures conditions d'enracinement des bouture herbacées de Chêne rouvre (Quercus petraea) et de Hêtre (Fagus silvatica). Ann. Sci. for., 34 (1), 1-16.

Courtoisier F., 1976. Etude des relations entre stations et qualité du bois de chêne en forêt de Bride et de Saint-Jean. Rapport de stage E.N.I.T.E.F., Station de Recherches sur la Qualité des Bois, C.N.R.F.

Garbaye J., Cornu D., Le Tacon F., 1975. Résultat d'un essai préliminaire sur le bouturage du chêne et du hêtre. Rev. for. fr., $\mathrm{n}^{\circ}$ 2, 135-140.

Garbaye J., Kazandian B., Le Tacon F., 1977. Développement des boutures racinées de Chêne rouvre (Quercus petraea). Premiers éléments d'une technique de production de plants. Ann. Sci. for., 34 (3), 245-260.

Kazandjian B., 1977. La multiplication végétative du chêne par bouturage. Rapport de stage E.N.I.T.E.F., Station de Recherches sur les Sols forestiers et la Fertilisation, C.N.R.F.

KeYLWerth R., 1954. Ein Beitrag zur qualitativen Zuwachsanalyse. Holz als Roh- u. Werkstoff, vol. $12, \mathrm{n}^{\circ} 3,77-83$.

Kleinschmit von J., Otto H., Sauer A., 1975. Möglichkeiten der züchterischen Verbesserung von Stiel- und Traubeneichen (Quercus robur und Quercus petraea). 1. Inventur der Eichensamenplantagen. Allgemeine Forst- und Jagdzeitung, 146, Helf. 9, 157-166.

Mourey J.M., 1979. Les chênes de la vallée de l'Ognon. Rapport de stage E.N.I.T.E.F., O.N.F. Besançon.

Nepveu G., 1973. Etude génétique de quelques qualités du bois de pin maritime. Corrélations avec des caractères de croissance et d'aptitude à l'élagage. Document à distribution limitée (Station de Recherches sur la Qualité des Bois), C.N.R.F., n ${ }^{\circ} 1973 / 5$.

Polge H., Illy G., 1968. Héritabilité de la densité du bois et corrélations avec la croissance étudiées à l'aide de tests non destructifs sur plants de pin maritime de quatre ans. Silvae Genet., 17, 5-6, 173-181.

Polge H., 1969. Influence de la fertilisation sur la qualité du bois de pin maritime. Ann. Sci. for., 26 (1), 45-64.

Polge H., Lemoine M., Deret E., 1977. Etude de la variabilité spécifique et infraspécifique de la structure juvénile du bois de chêne à l'aide d'un analyseur d'images. Ann. Sci. for., 34 (4), 285-292. 